\title{
Geomagnetic activity and local time dependence of the distribution of ultra low-frequency wave power in azimuthal wavenumbers, $m$
}

\author{
Theodore E. Sarris ${ }^{1,2}$ and Xinlin $\mathbf{L i}^{2}$ \\ ${ }^{1}$ Dept. of Electrical and Computer Engineering, Democritus University of Thrace, Xanthi, Greece \\ ${ }^{2}$ Laboratory for Atmospheric and Space Physics, Univ. of Colorado, Boulder, Colorado, USA \\ Correspondence to: Theodore E. Sarris (tsarris@ee.duth.gr)
}

Received: 24 December 2016 - Revised: 2 April 2017 - Accepted: 3 April 2017 - Published: 10 May 2017

\begin{abstract}
The azimuthal wavenumber $m$ of ultra lowfrequency (ULF) waves in the magnetosphere is a required parameter in the calculations of the diffusion rates of energetic electrons and protons in the magnetosphere, as electrons and protons of drift frequency $\omega_{\mathrm{d}}$ have been shown to radially diffuse due to resonant interaction with ULF waves of frequency $\omega=m \omega_{\mathrm{d}}$. However, there are difficulties in estimating $m$, due to lack of multipoint measurements. In this paper we use magnetic field measurements at geosynchronous orbit to calculate the cross-spectrogram power and phase differences between time series from magnetometer pairs. Subsequently, assuming that ULF waves of a certain frequency and $m$ would be observed with a certain phase difference between two azimuthally aligned magnetometers, the fraction of the total power in each phase difference range is calculated. As part of the analysis, both quiet-time and storm-time distributions of power per $m$ number are calculated, and it is shown that during active times, a smaller fraction of total power is confined to lower $m$ than during quiet times. It is also shown that in the dayside region, power is distributed mostly to the lowest azimuthal wavenumbers $m=1$ and 2, whereas on the nightside it is more equally distributed to all $m$ that can be resolved by the azimuthal separation between two spacecraft.
\end{abstract}

Keywords. Magnetospheric physics (energetic particles trapped; magnetospheric configuration and dynamics) space plasma physics (charged particle motion and acceleration)

\section{Introduction}

According to the basic concept of radial diffusion as described early on by Dungey (1954), as energetic particles drift around the Earth at a particular drift frequency $\omega_{\mathrm{d}}$ they can resonantly interact with electric and magnetic field oscillations of frequency $\omega=m \omega_{\mathrm{d}}$, where $m$ is the azimuthal wavenumber of the waves or else the number of azimuthal wavelengths that fit within a particle drift orbit. The exact determination of the azimuthal structure (and hence wavenumber $m$ ) of broadband ultra low-frequency (ULF) waves in the magnetosphere is not an easy task, as explained in detail, for example, by Waters et al. (2002). A way to calculate the azimuthal structure has been outlined by Holzworth and Mozer (1979), who applied a Fourier transform to measurements of the magnetic field spanning all local times and at different radial distances in $L$, followed by a Fourier transform in time. The result of the transform as described in their paper gives the power spectral density (PSD) of the ULF waves as a function of $m$ and frequency at different $L$. However, the exact computation of power at the various $m$ numbers according to this process requires multipoint measurements from multiple azimuthally aligned spacecraft across all local times and also across different $L$.

It is due to the scarcity of measurement points and the associated difficulties in calculating the azimuthal mode structure that in past radial diffusion simulations, it has been common to assume that all power is experienced by the particles through a single $m$ number. For example, in the work by Brautigam et al. (2005), it was assumed that all power is concentrated in $m=1$; this is similar in the work by Fei et al. (2006). In another example, Loto'aniu et al. (2006) estimated through ground measurements that the larger frac- 
tion of power was concentrated in $m=2$ during the particular event under investigation. The calculations in Loto'aniu et al. (2006) used phase difference calculation between the CARISMA (Canadian Array for Realtime Investigations of Magnetic Activity) and IMAGE (International Monitor for Auroral Geomagnetic Effects) as well as the SAMNET (UK Sub-Auroral Magnetometer Network) magnetometers. Perry et al. (2005) simulated the effect of ULF waves on the guiding centers of particles (including their bounce motion) through a 3-D model that they developed; in that study, only an azimuthal wavenumber $m=2$ was considered. Sarris et al. (2006) used a model of analytic pulse fields, distributing the power of compressional ULF oscillations with analytical expressions of their azimuthal modulation, enabling the study of the diffusive effects of different wavenumbers of ULF oscillations. In that study, the effect of both singlewavenumber compressional fluctuations of $m=1$ and also multiple-wavenumber compressional fluctuations of $m=1$ to $m=8$ on particle motion was investigated. For each of the two cases, the diffusion coefficient of magnetic diffusion $D_{L L}^{\mathrm{B}, \mathrm{Sym}}$ in a symmetric background field was determined from the radial transport of electrons that were traced in the simulation. In yet another study, Ozeke et al. (2012) assumed in their simulations that all power is concentrated in only one wavenumber, and the effects of values of $m=1$ and $m=10$ were tested in their diffusion coefficients.

In another example, Mann et al. (1998) used multi-satellite data and determined that an observed compressional waveguide mode had $m \leq 5$. Eriksson et al. (2006) studied the onset and development of a ULF pulsation that was excited by a storm sudden commencement (SSC) and, using measurements from the four Cluster spacecraft, estimated different azimuthal $m$ numbers at different harmonics: $m=10 \pm 3$ at the lower harmonic and several times higher for a higher harmonic. Zong et al. (2007) used observations of flux modulations of energetic electrons and ions and the deduced excitation of energetic electron drift resonance in order to estimate the prevailing $m$ number of toroidal ULF waves; the estimated wavenumber corresponded to $m \sim 10$. In a different approach, Tan et al. (2011) estimated the $m$ numbers of ULF waves near noon and midnight using the longitudinally distributed stations of the CARISMA and IMAGE magnetometer arrays, respectively, and found a considerable difference in the estimated $m$ values between noon and midnight, indicating the presence of azimuthal asymmetry of the wave modal components. They speculated that this asymmetry could be a "hidden" factor affecting the magnetospheric electron acceleration. The local time dependence of the power of ULF waves at different wavenumbers has also been shown through the study of Pilipenko et al. (2001), who compared two regions of broadband ULF intensification during substorms: one region was located in the early morning hours and the other one near dusk. This study has shown that nightside pulsations in the Pc5 band have higher $m$ numbers than typical dayside Pc5 pulsations.

Estimates of the mode structure can also be provided through physics-based models, such as magnetohydrodynamic (MHD) simulations, which capture ULF wave fluctuations across the entire magnetosphere as a response to solar wind disturbances; for example, Elkington et al. $(1999,2003)$ analyzed the structure of ULF waves during storm periods using the LFM (Lyon-Fedder-Mobarry) MHD model (Lyon et al., 2004). Similarly, Tu (2011) and Tu et al. (2009, 2012), obtained mode spectrum results and calculated the ULF wave azimuthal wavenumbers during a storm event from an analysis of the MHD simulation fields. Through this analysis it was estimated that the power at $m=1$ as calculated in the MHD was generally dominant, though sometimes if one assumes that all power comes from $m=1$, then the total power can be underestimated by a factor of up to 2. Interestingly, and related to the findings in this study, Tu et al. (2012) also found through the MHD simulation that, in general, the total power of the compressional component $B_{z}$ in $m=1$ is related primarily to solar wind dynamic pressure variations, while power in $m>1$ is related primarily to nightside geomagnetic activity.

In the following we use a cross-spectral and cross-phase analysis between time series of the ULF fluctuations of the total magnetic field in order to investigate the dominant $m$ number but primarily in order to estimate the distribution of power in the various $m$ numbers as a function of local time and its dependence on solar wind activity. In this study the focus is in the lowest wavenumbers, which are predominantly associated with the resonant interaction of ULF waves with energetic electrons and ions in the radiation belts. The phase differences in this technique are calculated between two measurement points that are azimuthally aligned, through which we can then extract information on the azimuthal wavelength of the waves. An overview of the technique that is used can be found in Sarris et al. (2009b, 2013). Furthermore, in Sarris (2014) it was demonstrated that the cross-phase technique could be used to provide an estimate of the distribution of the power of broadband ULF waves in the various low wavenumbers. In this paper, the principles of the same technique are applied to two prolonged periods: the first is a 10-day period of low solar and geomagnetic activity from 1 to 11 March 2013 during which Dst gradually increased from -70 to $0 \mathrm{nT}$ and during which gradual radial diffusion was observed by the Van Allen Probes in the radiation belts; the second is also a 10-day period from 7 to 17 October 2012, during which there is enhanced geomagnetic activity and associated ULF fluctuations. Through this analysis a local time dependence is demonstrated, with dayside seemingly favoring low- $m$ compressional ULF fluctuations, whereas on the nightside a more even distribution of power to all $m$ numbers is observed. Furthermore, through a comparison of the two events, it is found that the periods of lower geomagnetic activity generally favor the distribution of power in primarily 
low $m$ numbers, whereas intense geomagnetic activity favors a more even distribution of power to all $m$ numbers.

In the following, in Sect. 2 we describe the principles of the technique that is used herein to calculate the distribution of power in the various $m$ numbers. Subsequently, in Sect. 3 we apply this technique to observations of broadband ULF waves during the two 10-day periods mentioned above. In Sect. 4 we discuss the results of the study, focusing on their interpretation and on limitations of the applied technique, and in Sect. 5 we summarize the findings of this analysis and discuss ways to utilize them in more accurate determinations of the radial diffusion coefficients.

\section{Overview of the technique used to estimate the power per $m$ number}

The theoretical background for the application of crossspectral and cross-phase calculations to obtain the percentage of the distribution of total power in each wavenumber $m$ has been discussed in greater detail in Sarris et al. (2009b, 2013) and Sarris (2014). There, it was described that, for example, between two azimuthally displaced magnetometers that are separated by an angle $\Delta \varphi$, pulsations of azimuthal wavenumber $m=0$ in the magnetosphere would appear to have a zerodegree phase difference. Pulsations of $m=1$ are hemispheric oscillations, related to large-scale dayside or nightside pulsations; such pulsations would appear to have a $2 \pi$ modulation around the Earth regardless of frequency, and two azimuthally displaced magnetometers that are also separated by an angle $\Delta \varphi$ would measure a $\Delta \varphi$ phase difference between the two signals. Similarly, pulsations of $m=2$ correspond to oscillations of azimuthal extent equal to one quarter of the circumference of the Earth, often related to oscillations in the dawn and dusk side of the magnetosphere or even to dayside or nightside oscillations of small azimuthal extents; these would be characterized by a $4 \pi$ variation across all longitudes, whereas two azimuthally displaced magnetometers would appear to have a phase difference of $2 \cdot \Delta \varphi$. Similarly, $m=3$ corresponds to $3 \cdot \Delta \varphi$, etc. Thus, two magnetometers onboard two satellites azimuthally separated by $\Delta \varphi$ as above should be able to detect wavenumbers up to $2 \pi / \Delta \varphi$ by sorting the calculated phase differences between the ULF waves into bins of width $\Delta \varphi$.

For the calculation of phase differences between the two time series as a function of time and at all frequencies, we use the cross-wavelet transform (XWT) technique (e.g., Grinsted et al., 2004; Sarris et al., 2013; Sarris, 2014). The results of this calculation are wavelet and cross-wavelet power spectral density (XWT-PSD) in units of $\mathrm{nT}^{2} \mathrm{~Hz}^{-1}$ and also the calculated phase difference as a function of frequency and time.

In the following, for the two 10-day periods of October 2012 and March 2013, the phase differences are calculated between the magnetic field measurements of geosynchronous satellites GOES-13 and GOES-15, hereafter re-

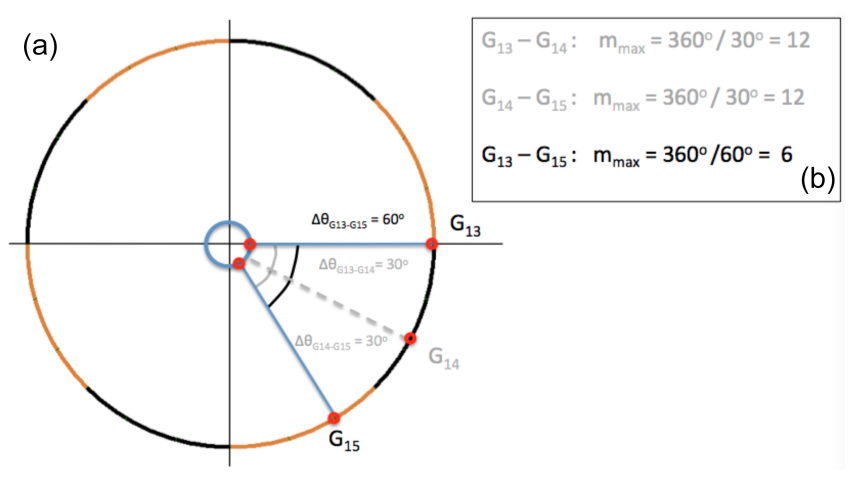

Figure 1. Relative azimuthal locations of three GOES satellites on October, 2012 (a), and maximum $m$ numbers that can be calculated by spacecraft pairs (b).

ferred to as $G_{13}$ and $G_{15}$, which are azimuthally separated by $\sim 60^{\circ}$ during the two events. The GOES (Geostationary Operational Environmental Satellite Program) satellites provide continuous monitoring from a geosynchronous orbit, with one satellite located over the east coast of the US at longitude $75^{\circ} \mathrm{W}\left(\mathrm{G}_{13}\right.$ in the two selected periods) and the other located over the west coast at longitude $135^{\circ} \mathrm{W}$ $\left(\mathrm{G}_{15}\right)$. Fortuitously, during the first 10-day period in October 2012 there are measurements available from a third satellite at longitude $\sim 105^{\circ} \mathrm{W}$, GOES-14 (hereafter referred to as $\mathrm{G}_{14}$ ), which was in on-orbit storage and on its way to an ultimate longitude of $75^{\circ} \mathrm{W}$, moving east at a rate of $0.9^{\circ}$ per day. The fortuitous magnetic field measurements from the third GOES satellite, $\mathrm{G}_{14}$, during the 10-day period in October 2012 enable us to perform the same analysis discussed above for higher wavenumbers, due to the closer distances between $\mathrm{G}_{13}-\mathrm{G}_{14}$ and $\mathrm{G}_{14}-\mathrm{G}_{15}$. At the same time, the additional two pairs of measurements enable us to confirm the $m$ number estimates from $\mathrm{G}_{13}$ and $\mathrm{G}_{15}$.

In Fig. 1 the relative locations of the three GOES satellites are plotted. According to the discussion above, the azimuthal separation between $G_{13}$ and $G_{15}$ allows the calculations of the distribution of ULF wave power in wavenumbers up to $m=360^{\circ} / 60^{\circ}=6$. In this analysis we present results that are limited to $m=2 \pi / \Delta \varphi-1$, or $m_{\max }=5$ from $\mathrm{G}_{13}$ and $\mathrm{G}_{15}$, as the global mode $m=0$ appears both at phase differences close to $0^{\circ}$ as well as at phase differences close to $360^{\circ}$ due to noise in the measurements. The location of $\mathrm{G}_{14}$ and its separation from $G_{13}$ and $G_{15}$ are marked in Fig. 1 and in the table therein in gray color; the separations in azimuth between the three pairs of GOES satellites and the maximum wavenumbers that can be calculated are also shown. In the same figure an example of the azimuthal extent of waves of wavenumber $m=4$ is shown, with four full wavelengths drawn as orange and black extents, indicating positive and negative phases. It is noted that satellites $\mathrm{G}_{13}$ and $\mathrm{G}_{15}$ would measure a phase difference $\Delta \varphi=m \cdot \Delta \varphi_{\mathrm{G}_{13}-\mathrm{G}_{15}}=240^{\circ}$ in this case. 


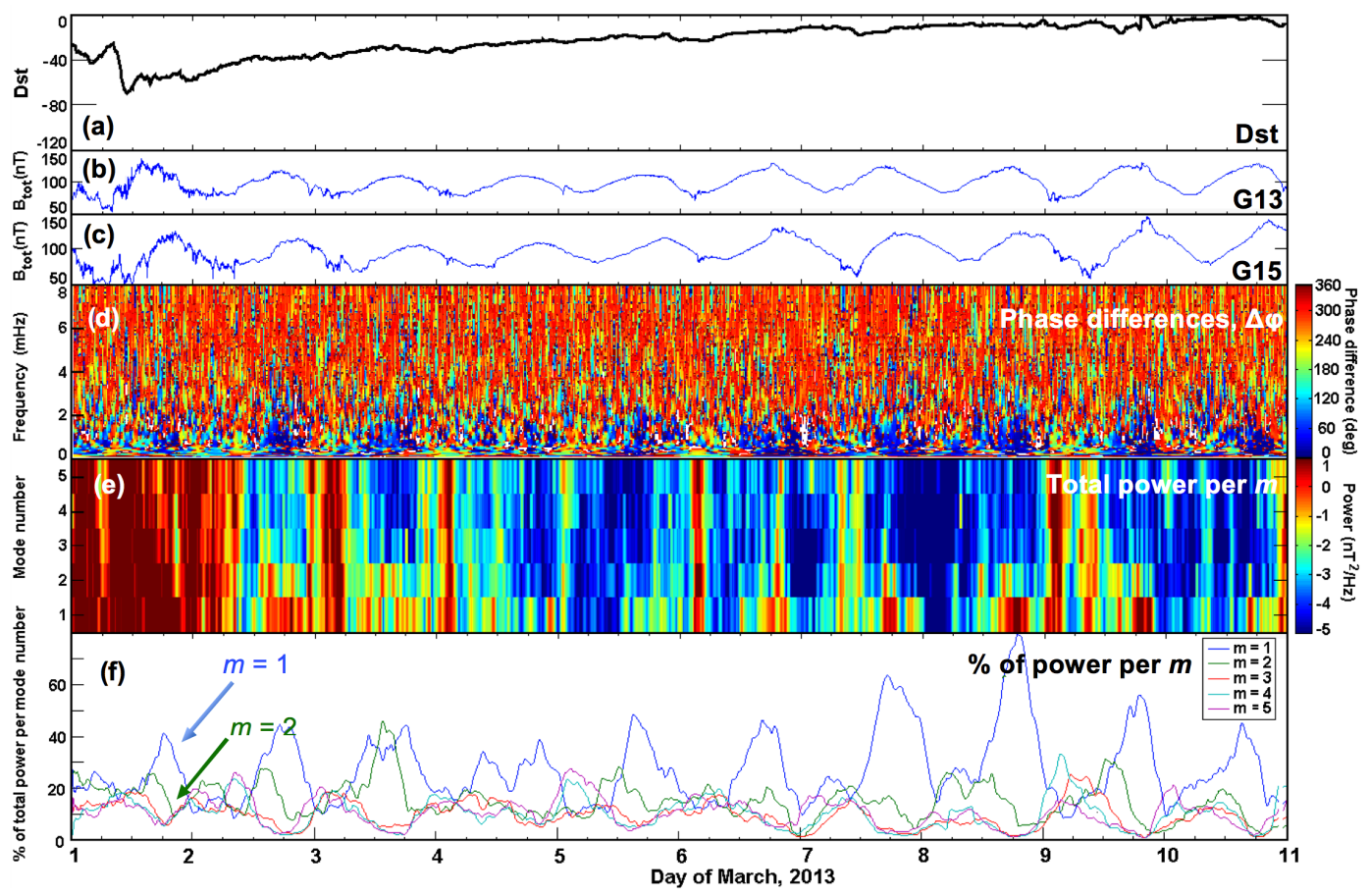

Figure 2. (a) Dst index during 1-11 March 2013; (b) GOES-13 and (c) GOES-15 total magnetic field; (d) calculations of the crossspectrogram phase differences between the two GOES time series as a function of frequency and time; (e) power per $m$ number calculations using cross-spectrogram calculations between GOES-13 and GOES-15; (f) fraction of the total power in each of the wavenumbers $m=1$ to 5 .

\section{Azimuthal wavelengths at different levels of geomagnetic activity}

We apply the technique discussed above to two distinct time periods with very different characteristics: the first is a 10day period from 1 to 11 March 2013, which has been described as a remarkably clear example of inward radial transport of energetic electrons (Baker et al., 2014), as no other processes appear to be at play during this time. During this time an initial enhancement in electron fluxes up to $5.6 \mathrm{MeV}$ was measured on 1 March at larger $L$ by the Relativistic Electron-Proton Telescope (REPT) instrument (Baker et al., 2012) onboard the Van Allen Probes twin-spacecraft mission. The initial enhancement of 1 March was associated with a fast speed stream and was followed by inward transport over the next 2 weeks, such that by 17 March the peak in electron fluxes was observed near $L=4.0$. These features make this event a prime example of continuous and gradual inward radial diffusion (e.g., Schulz and Lanzerotti, 1974).

The second interval that was investigated for comparison is a 10-day period from 7 to 17 October 2012, a period of enhanced geomagnetic activity, including three consecutive storms that impacted the Earth's magnetosphere. During this time high-energy electron fluxes were enhanced deep inside the magnetosphere, down to $L=4.0$. This event has been studied in further detail in Reeves et al. (2013), where the electron enhancement has been described as a clear illustra- tion of a local acceleration mechanism for relativistic electron acceleration in the heart of the outer Van Allen belt. In particular, the 8-9 October storm not only shows a remarkable enhancement of radiation belt electrons but also a fast electron dropout preceding the enhancement, as discussed in $\mathrm{Tu}$ et al. (2014). Magnetopause shadowing combined with enhanced outward radial diffusion could play an important role in the observed radiation belt dropout. Thus, it is important to be able to model the ULF waves accurately during this time, including their azimuthal wavenumber, in order to assess the potential importance of ULF wave-driven radial diffusion in the electron dropout during this period in addition to the local acceleration.

Measurements and analysis results for the phase differences between the magnetic field time series for the event of 1-11 March 2013 when geomagnetic activity decreases and Dst gradually approaches 0 are shown in Fig. 2, whereas results for the event of 7-17 October 2012 of enhanced geomagnetic activity are shown in Fig. 3. The sequence of the panels in the two figures follow the same format: In panel (a) of the two figures the Dst index over the two 10-day periods is shown. In panels (b) and (c), measurements of the total magnetic field are shown from spacecraft $G_{13}$ and $G_{15}$, respectively; the peaks of the diurnal variation of the total magnetic field correspond to measurements that are made near local noon. In panel (d) the estimated phase differences 


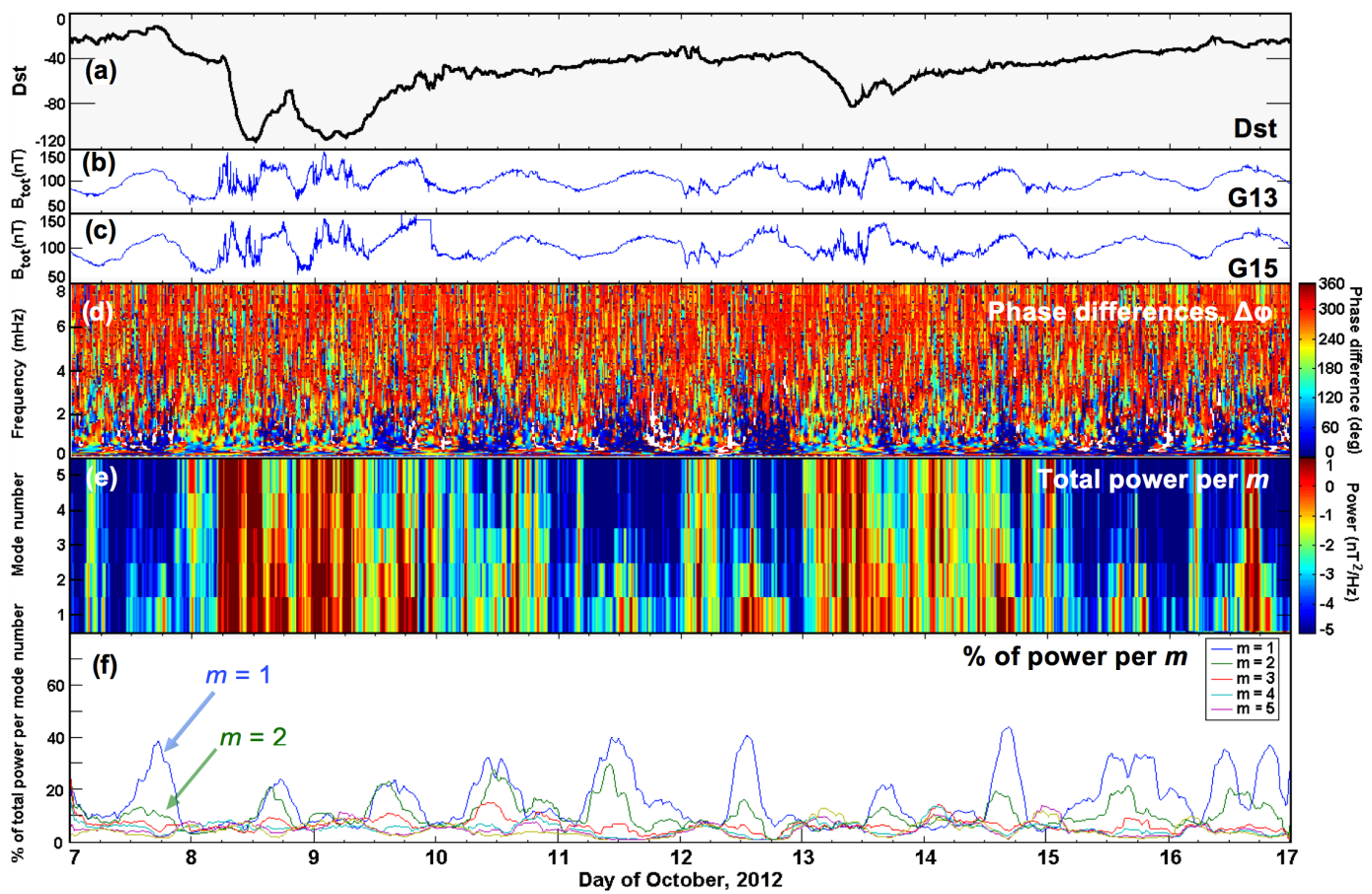

Figure 3. (a) Dst index during 7-17 October 2012; (b) GOES-13 and (c) GOES-15 total magnetic field; (d) cross-spectrogram phase differences between (b) and (c); (e) power per $m$ number; (f) fraction of the total power in each of the wavenumbers $m=1$ to 5 .

between measurements at $\mathrm{G}_{13}$ and $\mathrm{G}_{15}$ are plotted as a function of frequency, whereas in panel (e) the cross-spectrogram power between the two spacecraft is binned according to $m$, following the methodology described in Sarris (2014). In panel (f) the percentage of power in a particular $m$ number over power in all $m$ numbers from 1 to 5 is plotted as a function of time, with each $m$ number plotted in a different color, as shown in the inbox. The blue line corresponding to $m=1$ and the green line corresponding to $m=2$ are indicated with labels and arrows for easier reference.

In order to calculate the cross-spectrogram power as a function of both time and $m$, as shown in panel (e) of Figs. 2 and 3 for the two events, respectively, the following methodology is applied: first, the XWT is calculated for the continuous wavelet transforms (CWTs) of each of the two time series, as described in, e.g., Torrence and Compo (1998) and Torrence and Webster (1999) and, for an application to magnetometer data, in Sarris et al. (2013) and Sarris (2014). The XWT thus indicates the times and frequencies with common power at the two spacecraft. The cross-spectrogram power between the two spacecraft is binned as a function of $m$ number and time following the power-per-phase difference technique of Sarris (2014) as is briefly described below.

Waves of azimuthal wavenumber $m=0$ (global oscillations of the magnetosphere) would appear through crosswavelet analysis as having a phase difference of $0^{\circ}$ between the two satellites $\mathrm{G}_{13}$ and $\mathrm{G}_{15}$. Furthermore, as $\mathrm{G}_{13}$ and $\mathrm{G}_{15}$ are separated in azimuth by $60^{\circ}$, waves of $m=1$ would ap- pear to have a phase difference of $60^{\circ}$. Hence, in each time bin we sum all the power that corresponds to phase differences between 0 and $60^{\circ}$, which we attribute to the total power in the range of $m=0$ to 1 . Similarly, if all power at phase differences from 60 and $120^{\circ}$ is summed, this will correspond to the power in the range of $m=1$ to 2 , etc. The separation in azimuth between $\mathrm{G}_{13}$ and $\mathrm{G}_{15}$ enables the estimation of the power distribution from $m=0$ up to $m_{\max }=\operatorname{INT}\left(360^{\circ} / 60^{\circ}\right)=6$, where INT indicates the integer number of the ratio in parentheses. It has been observed, however, that azimuthal wavenumbers up to $\left(m_{\max }-1\right)$, or up to $m=5$ in this case, can actually be investigated as phase differences close to $0^{\circ}$ are misinterpreted as phase differences close to $360^{\circ}$, potentially due to noise in the measurements but also due to aliasing effects (see discussion below). The total cross-spectrogram power binned per phase difference as described above is plotted as a function of $m$ number and time in panel (e) of both Figs. 2 and 3. Thus, in Fig. 2e we can see that intense fluctuations can be observed across all $m$ numbers on 1 March 2013, during the drop of Dst to approximately -70 , whereas intense fluctuations can also be seen across all $m$ numbers around the midnight or pre-midnight region on most of the 10 days of the event, indicating the appearance of more intense ULF waves in the magnetotail than on the morning side. Correspondingly, in Fig. 3e we can see that intense fluctuations can be observed across all $m$ numbers on 8 October 2012, during and after the drop of Dst to 


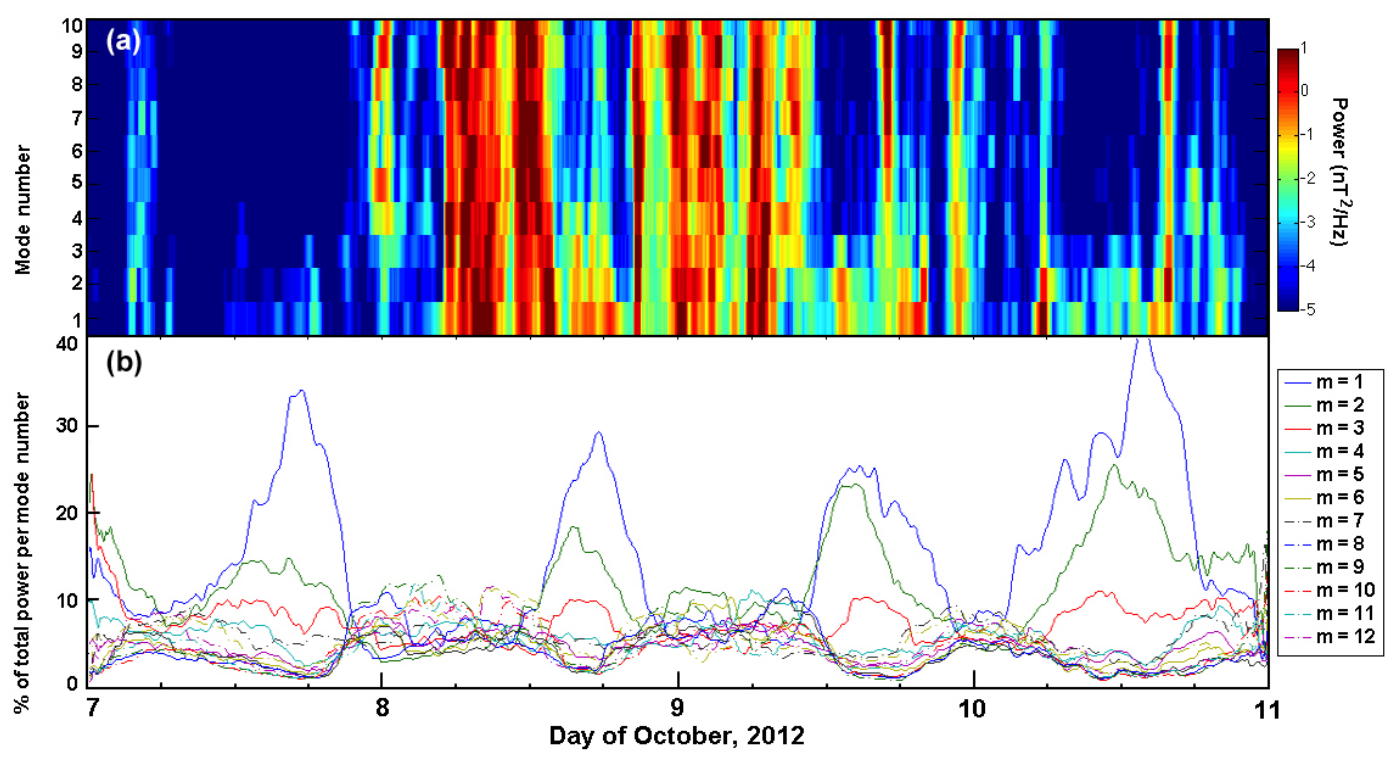

Figure 4. (a) Power per $m$ number calculations for the first 4 days of Fig. 3, using cross-spectrogram calculations between GOES-14 and GOES-15; (b) fraction of power in one of the wavenumbers $m=1$ to 12 over total power. Lower wavenumbers $m=1$ to 6 are plotted with solid lines, and higher wavenumbers $m=7$ to 12 are plotted with dashed-dotted lines.

approximately -120 , as well as throughout the entire 10-day period.

In Figs. $2 \mathrm{f}$ and $3 \mathrm{f}$ we plot the fraction of the total ULF wave power at the first wavenumbers from $m=1$ through $m=5$ : the fraction of total power in $m=1$ is plotted with a blue line, $m=2$ with a green line, $m=3$ with a red line, etc, as marked. From these plots we can observe a strong diurnal variation in the percentage of total power in each $m$ number, with the dayside favoring a larger fraction of power in lower $m$ numbers $m=1$ and $m=2$, reaching from 40 up to $70 \%$ in $m=1$ for the more geomagnetically quiet event of Fig. $2 \mathrm{f}$ and from 20 to $40 \%$ in $m=1$ for the more geomagnetically active event of Fig. 3f. A correlation can be observed between Dst and the percentage of the power of lower $m$ numbers, primarily on the dayside, with a Dst index closer to 0 corresponding to a higher percentage of total power in lower $m$ numbers. Furthermore, the overall distribution of power in the lower mode numbers is higher for the geomagnetically quiet event of Fig. 2f than for the active event of Fig. 3f. In the nightside magnetosphere, power seems to be equally divided amongst the first five $m$ numbers for both events. This is further discussed in the next section.

One should keep in mind that an aliasing can arise if, for example, a measured phase difference $\Delta \varphi$ that is attributed to an assumed wavenumber $m$ actually corresponds to a phase difference $2 \pi n+\Delta \varphi$, where $n>2 \pi / \Delta \lambda$ and $\Delta \lambda$ is the angular separation between the measurement points. In order to confirm the results presented above and to investigate the possibility of spatial aliasing, as described in, e.g., Chisham and Mann (1999), we apply the same analysis using different pairs of satellites for the period of 8 to 12 October 2012, when measurements from a third GOES satellite, $G_{14}$, were available. $G_{14}$ was located between $G_{13}$ and $G_{15}$, enabling the same analysis from pair $\mathrm{G}_{13}$ and $\mathrm{G}_{14}$, as well as from pair $\mathrm{G}_{14}$ and $\mathrm{G}_{15}$, with a closer azimuthal separation, which makes it possible to resolve the distribution of power to even higher $m$ numbers, as shown in Fig. 1. The results after applying the same analysis are presented in Fig. 4. Thus, in Fig. 4a the distribution of power in $m$ is shown for wavenumbers up to $m=12$, whereas in Fig. $4 \mathrm{~b}$ the fraction of total power in each $m$ number range is shown.

In comparing the fractions of power in $m$ between Fig. 3 (where a $60^{\circ}$ separation exists between $\mathrm{G}_{13}$ and $\mathrm{G}_{15}$ ) and Fig. 4 (where a $30^{\circ}$ separation exists between $\mathrm{G}_{14}$ and $\mathrm{G}_{15}$ and where the power in twice as many wavenumbers can be approximated), we can see that for each $m$ fraction line similar fractions are observed between the two figures: for example, in the dayside region between 7 and 8 October, $m=1$ has a peak of $\sim 37 \%$ of total power as calculated from $\mathrm{G}_{13}$ and $\mathrm{G}_{15}$ (Fig. 3) and $\sim 35 \%$ as calculated from $\mathrm{G}_{14}$ and $\mathrm{G}_{15}$ (Fig. 4); $m=2$ has a peak of $\sim 13 \%$ from $\mathrm{G}_{13}$ and $\mathrm{G}_{15}$ (Fig. 3) and $\sim 15 \%$ from $\mathrm{G}_{14}$ and $\mathrm{G}_{15}$ (Fig. 4); and the higher $m$ numbers range between 1 and $10 \%$ as calculated from both the $\mathrm{G}_{13}$ and $\mathrm{G}_{15}$ and the $\mathrm{G}_{14}$ and $\mathrm{G}_{15}$ pairs. On the nightside, for the same day, power in all $m$ numbers ranges between 5 and $10 \%$ as calculated from $\mathrm{G}_{13}$ and $\mathrm{G}_{15}$ (Fig. 3) and between 4 and $13 \%$ as calculated from $\mathrm{G}_{14}$ and $\mathrm{G}_{15}$ (Fig. 4). Thus, we can see that the results from pair $\mathrm{G}_{13}$ and $\mathrm{G}_{14}$ confirm the results from pair $\mathrm{G}_{13}$ and $\mathrm{G}_{15}$, as the power distribution for wavenumbers up to $m=5$ is about the same as shown in Fig. 3, whereas much less power is observed in wavenumbers $m>5$ on the dayside; similarly, 
as above, power is distributed to all $m$ numbers in the local midnight sectors. We can thus conclude that the aliasing effect is not significant and that the methodology does not move considerable amounts of total wave power associated with high- $m$ numbers to waves with low- $m$ numbers.

\section{Discussion}

Through the above analysis, the following key conclusions can be reached: Firstly, the assumption often made in radial diffusion models that all ULF wave power can be attributed primarily to one azimuthal wavenumber is not always an accurate representation. Furthermore, ULF wave power is not distributed at the various wavenumbers in a similar fashion across all local times, but instead there is a local time dependence in this distribution, with dayside favoring the distribution of power in the lower $m$ numbers. Finally, the ULF wave power distribution in the various $m$ numbers has geomagnetic activity dependence, with higher activity favoring the distribution of power to higher $m$ numbers, corresponding to smaller azimuthal-scale structures in the magnetosphere.

Regarding the local time dependence of the power per $m$ number, patterns of the distribution of power at the various wavenumbers across different local times are clearly demonstrated, with power generally reaching a local peak in the lower $m$ numbers (corresponding to larger wavelengths) $m=1$ and 2 around noon, whereas power appears to be equally distributed among $m$ numbers around the midnight region. The local peak in the noon side could be attributed to ULF waves that are caused by, among other excitation mechanisms, large-scale compressional fluctuations of the dayside magnetopause and which are expected to launch compressional perturbations and broadband waves of mainly low wavenumbers primarily in the dayside magnetosphere. On the other hand, the ducting of these ULF waves from the dayside to the nightside through the magnetosphere flanks potentially involves the breaking of these waves to smaller wavelengths, through reflections between the flank magnetopause and the plasmasphere; at the same time, magnetotail flapping and substorms also contribute to smaller-scale fluctuations in the midnight region, which can be expected to translate to enhanced power in the higher $m$ numbers.

Regarding the geomagnetic activity dependence of the power distribution in $m$, we note in Fig. $2 \mathrm{f}$ that the percentage of power going to $m=1$ and 2 generally increases for increasing values of the Dst index (decreasing activity), indicating that during quiet times a larger fraction of total power is confined to lower $m$ than during active times. Thus, on the first day of the event, where Dst is lower than $-40 \mathrm{nT}$, the peak of $m=1$ on the dayside reaches up to $\sim 40 \%$, whereas on the following days, where Dst increases towards 0 , the peak of power at $m=1$ also increases, reaching values higher than $60 \%$ for Dst higher than $-10 \mathrm{nT}$. This trend is confirmed in the active 10-day period of October 2012: in
Fig. $3 \mathrm{f}$ when Dst is greater than $-40 \mathrm{nT}$ the peak in power at $m=1$ reaches values greater than $40 \%$, whereas during each of the three storm times and the corresponding drops in Dst, the peaks in power are below $20 \%$. A potential explanation of this observation is that active times are potentially associated with more turbulent phenomena together with enhanced shear in the magnetosphere flanks, all of which potentially favor smaller wavelength phenomena and hence larger wavenumbers $m$. This interpretation needs to be further investigated through comparisons of spacecraft observations with MHD model results.

Together with the geomagnetic activity (Dst) dependence of the power distribution in $m$, we have investigated its correlation with solar wind parameters such as solar wind velocity and dynamic pressure (plots not included). These parameters do not show such a direct correlation with the power distribution in $m$ as Dst, in particular for the times of high activity. However, the dependence on various geomagnetic and solar wind parameters needs to be investigated quantitatively through a statistical, long-term study.

In the above analysis we note that the methodology that was used considers only eastward-propagating waves, as their resonance with drifting electrons make them relevant to the electron diffusion coefficient. This does not mean that eastward-propagating waves have to be dominant, and there is no implied assumption about a preferential propagating direction: for example, surface waves or the Kelvin-Helmholtz instability on the magnetopause are common sources of compressional waves, and their propagation direction depends on local time. Furthermore, compressional variations in the magnetic field such as those examined herein can be decomposed into a westward-propagating and an eastward-propagating wave; however, only eastwardpropagating waves will resonantly interact with eastwarddrifting electrons leading to enhanced radial diffusion. Thus, the phase differences of waves propagating eastward from $\mathrm{G}_{15}$ to $\mathrm{G}_{13}$, assumed here as positive phase differences, have been considered. In the case of protons, the same process needs to be applied for phase difference calculations between $\mathrm{G}_{13}$ and $\mathrm{G}_{15}$, which would result in negative phase differences. Potential implications to the total wave power for calculating the radial diffusion equation when considering only eastward- vs. westward-propagating waves instead of the total wave power have been described in Sarris et al. (2006).

The fact that the results from greatly different azimuthal separations between spacecraft are comparable for the same event is evidence that the spectral aliasing effect due to under-sampling is not very large, at least in the events investigated herein. We note that a lower power can be expected in the lowest $m$ numbers for a closer separation between the two spacecraft, when higher $m$ numbers are identifiable. This is what is observed for $m=1$ when comparing Figs. 3 and 4, but the percentage of difference is rather small and does not alter the key results of this study, namely the estimates of the 
solar wind and local time dependence of the distribution of power in $m$.

It should be pointed out that the measured phase differences and the propagation characteristics that are described above could not necessarily be considered as propagation of well-coherent waves. In reality, well-coherent Pc5 waves at two spacecraft azimuthally separated by as much as $60^{\circ}$ for a prolonged period are not a very common phenomenon (for an exception to this, see, e.g., the prolonged event described in Sarris et al., 2009a). However, for the purposes of investigating the effects of ULF waves on the radial diffusion of energetic electrons, the broadband (in azimuthal wavelengths) spatial structure of ULF waves is decomposed in these prevailing individual azimuthal wavenumbers in the same way as the spectrally broadband ULF waves are decomposed into a series of frequencies in order to identify the components which are resonant with each drift period.

A particular implication of determining the power in azimuthal wavenumbers for noncoherent waves is evident in the midnight regions of Figs. 3 and 4, where the wave power appears to be distributed over all $m$ numbers up to the upper limit that can be analyzed, without the appearance of a prevailing wavenumber: if the waves over these regions are not coherent and the two satellites observe uncorrelated disturbances, then a cross-phase analysis of the two time series would detect to some extent random phase differences across the spectrum and the method used in this study would allocate wave power across all $m$ numbers, up to the limit that can be resolved based on the given spacecraft separation. However, even in this case, the fact that low wavenumbers do not prevail in terms of power at these local times together with the equal distribution of power across all wavenumbers constitute critical information that can be used in attributing power in $m$ when calculating the radial diffusion coefficients. A further investigation needs to be carried out for cases in which more geosynchronous spacecraft are simultaneously available, in order to investigate the range of variability of the distribution to the various numbers in the case of noncoherent waves.

\section{Summary and conclusions}

In this paper an XWT phase difference technique has been used to provide insight into as well as more accurate estimates of the distribution of power to each of the lowest azimuthal wavenumbers of magnetospheric broadband ULF waves, to the extent permitted by the azimuthal separation of the measurement points. These more accurate estimates of the mode structure of the ULF waves are critical in modeling efforts of radial diffusion, which currently assume single$m$ structures. In this study the emphasis was placed on the compressional component of magnetic field ULF perturbations; however, the electric field ULF variations, manifested through the electrostatic diffusion coefficient, and toroidal waves are also critical in radial diffusion processes. Thus, the analysis performed as part of this study needs to be expanded to include the electric field and horizontal magnetic field components as well.

This technique has been applied to two greatly different periods: the period between 1 and 11 March 2013, which initiated with a solar wind stream but had gradually diminishing geomagnetic activity with Dst gradually approaching 0 , and a more active time period between 7 and 17 October 2012, when three consecutive storms impacted the Earth. Using measurements from pairs of geosynchronous satellites, we obtained estimates of the distribution of power in the lowest five azimuthal wavenumbers during these two 10day periods. The technique described herein was able to capture a local time dependence of the distribution of power per $m$, with dayside generally favoring lower wavenumbers. Furthermore, the technique described herein was able to capture a dependence on geomagnetic activity, with higher Dst (lower geomagnetic activity) generally favoring lower wavenumbers.

One limitation of the technique that has been used herein is that the results demonstrating the local time and geomagnetic activity dependence of the azimuthal structure of ULF waves are limited to geosynchronous orbit; thus, the application of these results to models of radial diffusion will need to make the assumption that similar distributions of power in $m$ apply to all $L$, the validity of which needs to be further investigated. Secondly, there is a spatiotemporal ambiguity in the results, as the spacecraft pair moves across local times, while there are ongoing magnetospheric changes; the use of more than one pair of geosynchronous spacecraft, whenever available, can provide input into the criticality of this limitation. A third limitation arises from the finite azimuthal separation of the magnetometer pairs, setting a limit on the maximum wavenumber that can be measured, potentially resulting in artificially high fractions in the lower $m$, which should potentially be distributed to wavenumbers that cannot be resolved based on the particular configurations; however, the lowest $m$ numbers often contain most of the ULF wave power, in particular during active times. A fourth limitation involves the fact that there is a $2 \pi$ ambiguity when resolving the phase differences between two time series, meaning that a phase difference of $360^{\circ}+\Delta \varphi$ that corresponds to a wavenumber higher than $m_{\max }$ would be detected as $\Delta \varphi$; however, the comparison between the results from two different pairs of geosynchronous satellites, as discussed above and shown in Figs. 3 and 4, shows little difference in the power per $m$ for spacecraft separations of 60 and $30^{\circ}$. A statistical study for a number of similar cases with more than one satellite pair can provide input on when this methodology can be safely applied.

In conclusion, despite the limitations discussed above, the methodology presented in this paper reveals details of the distribution of power in the various wavenumbers that are significantly more accurate than the oversimplifying assump- 
tion that all power is attributed to one wavenumber, which is most commonly encountered in radial diffusion studies and simulations. The ability of the proposed technique to provide a qualitative and quantitative estimate of how the ULF wave power is distributed in the various wavenumbers $m$ could prove to be a very important addition in models that use radial diffusion coefficients to model the evolution of particle populations, which could improve the prediction capabilities and modeling of the radiation belt particles. In particular, the reported magnetospheric activity dependence of the distribution of ULF wave power in $m$ may provide insight into the sequence of events and the time history of the energization of relativistic electrons during a storm vs. during quiet times.

Data availability. GOES magnetometer data are available at http: //satdat.ngdc.noaa.gov/sem/goes/.

Competing interests. The authors declare that they have no conflict of interest.

Acknowledgements. We thank NOAA and Howard Singer for making available GOES magnetometer data. This study was supported by NASA contract NAS5-02099 (THEMIS mission), NASA grants NNX15AF56G and NNX12AG37G and NSF grant AGS 1131869. This research has also been co-financed by the European Union (European Social Fund - ESF) and Greek national funds through the Operational Program "Education and Lifelong Learning" of the National Strategic Reference Framework (NSRF) - Research Funding Program: Thales. Investing in knowledge society through the European Social Fund.

The topical editor, E. Roussos, thanks T. Yeoman and one anonymous referee for help in evaluating this paper.

\section{References}

Baker, D. N., Kanekal, S. G., Hoxie, V. C., Batiste, S., Bolton, M., Li, X., Elkington, S. R., Monk, S., Reukauf, R., Steg, S., Westfall, J., Belting, C., Bolton, B., Braun, D., Cervelli, B., Hubbell, K., Kien, M., Knappmiller, S., Wade, S., Lamprecht, B., Stevens, K., Wallace, J., Yehle, A., Spence, H. E., and Friedel, R.: The Relativistic Electron-Proton Telescope (REPT) instrument on board the Radiation Belt Storm Probes (RBSP) spacecraft: Characterization of Earth's radiation belt high-energy particle populations, Space Sci. Rev., 179, 337, doi:10.1007/s11214-012-99509, 2012.

Baker, D. N., Jaynes, A. N., Li, X., Henderson, M. G., Kanekal, S. G., Reeves, G. D., Spence, H. E., Claudepierre, S. G., Fennell, J. F., Hudson, M. K., Thorne, R. M., Foster, J. C., Erickson, P. J., Malaspina, D. M., Wygant, J. R., Boyd, A., Kletzing, C. A., Drozdov, A., and Shprits, Y. Y.: Gradual diffusion and punctuated phase space density enhancements of highly relativistic electrons: Van Allen Probes observations, Geophys. Res. Lett., 41, 1351-1358, doi:10.1002/2013GL058942, 2014.
Brautigam, D. H., Ginet, G. P., Albert, J. M., Wygant, J. R., Rowland, D. E., Ling, A., and Bass, J.: CRRES electric field power spectra and radial diffusion coefficients, J. Geophys. Res., 110, A02214, doi:10.1029/2004JA010612, 2005.

Chisham, G. and Mann, I. R.: A Pc5 ULF wave with large azimuthal wavenumber observed within the morning sector plasmasphere by Sub-Auroral Magnetometer Network. J. Geophys. Res., 104, 14717-14727, doi:10.1029/1999JA900147, 1999.

Dungey, J. W.: Electrodynamics of the outer atmosphere, Rep. 69, Ions. Res. Lab., Pa. Sate Univ., University Park, Pa, 1954.

Elkington, S. R., Hudson, M. K., and Chan, A. A.: Acceleration of relativistic electrons via drift resonant interaction with toroidalmode Pc-5 ULF oscillations, Geophys. Res. Lett., 26, 3273, doi:10.1029/1999GL003659, 1999.

Elkington, S. R., Hudson, M. K., and Chan, A. A.: Resonant acceleration and diffusion of outer zone electrons in a asymmetric geomagnetic field, J. Geophys. Res., 108, 1116, doi:10.1029/2001JA009202, 2003.

Eriksson, P. T. I., Blomberg, L. G., Schaefer, S., and Glassmeier, K.H.: On the excitation of ULF waves by solar wind pressure enhancements, Ann. Geophys., 24, 3161-3172, doi:10.5194/angeo24-3161-2006, 2006.

Fei, Y., Chan, A. A., Elkington, S. R., and Wiltberger, M.: Radial diffusion and MHD-particle simulations of relativistic electron transport by ULF waves in the September 1998 storm, J. Geophys. Res., 111, A12209, doi:10.1029/2005JA011211, 2006.

Grinsted, A., Moore, J. C., and Jevrejeva, S.: Application of the cross wavelet transform and wavelet coherence to geophysical time series, Nonlin. Processes Geophys., 11, 561-566, doi:10.5194/npg-11-561-2004, 2004.

Holzworth, R. H. and Mozer, F. S.: Direct evaluation of the radial diffusion coefficient near $L=6$ due to electric field fluctuations, J. Geophys. Res., 84, 2559, doi:10.1029/JA084iA06p02559, 1979.

Loto'aniu, T. M., Mann, I. R., Ozeke, L. G., Chan, A. A., Dent, Z. C., and Milling, D. K.: Radial diffusion of relativistic electrons into the radiation belt slot region during the $2003 \mathrm{Hal}-$ loween geomagnetic storms, J. Geophys. Res., 111, A04218, doi:10.1029/2005JA011355, 2006.

Lyon, J. G., Fedder, J. A., and Mobarry, C. M.: The LyonFedder-Mobarry (LFM) global MHD magnetospheric simulation code, J. Atmos. Sol.-Terr. Phy., 66, 1333, doi:10.1016/j.jastp.2004.03.020, 2004.

Mann, I. R., Chisham, G., and Bale, S. D.: Multisatellite and ground-based observations of a tailward propagating Pc5 magnetospheric waveguide mode, J. Geophys. Res., 103, 4657-4669, 1998.

Ozeke, L. G., Mann, I. R., Murphy, K. R., Rae, I. J., Milling, D. K., Elkington, S. R., Chan, A. A., and Singer, H. J.: ULF wave derived radiation belt radial diffusion coefficients, J. Geophys. Res., 117, A04221, doi:10.1029/2011JA017335, 2012.

Perry, K. L., Hudson, M. K., and Elkington, S. R.: Incorporating spectral characteristics of Pc5 waves into three-dimensional radiation belt modeling and the diffusion of relativistic electrons, J. Geophys. Res., 110, A03215, doi:10.1029/2004JA010760, 2005.

Pilipenko, V., Kleimenova, A. N., Kozyreva, O., Engebretson, M., and Rasmussen, O.: Long-period magnetic activity during the May 15, 1997 storm, J. Atmos. Sol.-Terr. Phy., 63, 489-501, 2001. 
Reeves, G. D., Spence, H. E., Henderson, M. G., Morley, S. K., Friedel, R. H. W., Funsten, H. O., Baker, D. N., Kanekal, S. G., Blake, J. B., Fennell, J. F., Claudepierre, S. G., Thorne, R. M., Turner, D. L., Kletzing, C. A., Kurth, W. S., Larsen, B. A., and Niehof, J. T.: Electron acceleration in the heart of the Van Allen radiation belts, Science, 341, 991-994, 2013.

Sarris, T. E.: Estimates of the power per mode number of broadband ULF waves at geosynchronous orbit, J. Geophys. Res.-Space, 119, doi:10.1002/2013JA019238, 2014.

Sarris, T., Li, X., and Temerin, M.: Simulating radial diffusion of energetic $(\mathrm{MeV})$ electrons through a model of fluctuating electric and magnetic fields, Ann. Geophys., 24, 2583-2598, doi:10.5194/angeo-24-2583-2006, 2006.

Sarris, T., Li, X., and Singer, H. J.: A long-duration narrowband Pc5 pulsation, J. Geophys. Res., 114, A01213, doi:10.1029/2007JA012660, 2009a.

Sarris, T. E., Liu, W., Kabin, K., Li, X., Elkington, S. R., Ergun, R., Rankin, R., Angelopoulos, V., Bonnell, J., Glassmeier, K. H., and Auster, U.: Characterization of ULF pulsations by THEMIS, Geophys. Res. Lett., 36, L04104, doi:10.1029/2008GL036732, 2009b.

Sarris, T. E., Li, X., Liu, W., Argyriadis, E., Boudouridis, A., and Ergun, R.: Mode number calculations of ULF fieldline resonances using ground magnetometers and THEMIS measurements, J. Geophys. Res.-Space, 118, 6986-6997, doi:10.1002/2012JA018307, 2013.

Schulz, M. and Lanzerotti, L. J.: Particle Diffusion in the Radiation Belts, Springer-Verlag, New York, 1974.

Tan, L. C., Shao, X., Sharma, A. S., and Fung, S. F.: Relativistic electron acceleration by compressional-mode ULF waves: Evidence from correlated Cluster, Los Alamos National Laboratory spacecraft, and ground-based magnetometer measurements, J. Geophys. Res., 116, A07226, doi:10.1029/2010JA016226, 2011.
Torrence, C. and Compo, G. P.: A practical guide to wavelet analysis, B. Am. Meteorol. Soc., 79, 61-78, 1998.

Torrence, C. and Webster, P. G.: Interdecadal changes in the ENSOMonsoon System, J. Climate, 12, 2679-2690, 1999.

Tu, W.: Modeling Earth's Outer Radiation Belt Electron Dynamics - Radial Diffusion, Heating, and Loss, PhD Thesis, University of Colorado at Boulder, 2011.

Tu, W., Li, X., Chen, Y., Reeves, G. D., and Temerin, M.: Stormdependent radiation belt electron dynamics, J. Geophys. Res., 114, A02217, doi:10.1029/2008JA013480, 2009.

Tu, W., Elkington, S. R., Li, X., Liu, W., and Bonnell, J.: Quantifying radial diffusion coefficients of radiation belt electrons based on global MHD simulation and spacecraft measurements, J. Geophys. Res., 117, A10210, doi:10.1029/2012JA017901, 2012.

Tu, W., Cunningham, G. S., Chen, Y., Morley, S. K., Reeves, G. D., Blake, J. B., Baker D. N., and Spence, H.: Event-specific chorus wave and electron seed population models in DREAM3D using the Van Allen Probes, Geophys. Res. Lett., 41, 1359-1366, doi:10.1002/2013GL058819, 2014.

Waters, C. L., Takahashi, K., Lee, D.-H., and Anderson, B. J.: Detection of ultralow-frequency cavity modes using spacecraft data, J. Geophys. Res., 107, 1284, doi:10.1029/2001JA000224, 2002.

Zong, Q.-G., Zhou, X.-Z., Li, X., Song, P., Fu, S. Y., Baker, D. N., Pu, Z. Y., Fritz, T. A., Daly, P., Balogh, A., and Reme, H.: Ultra-low frequency modulation of energetic particles in the dayside magnetosphere, Geophys. Res. Lett., 34, L12105, doi:10.1029/2007GL029915, 2007. 